\title{
The Role of Acupuncture Improving Cognitive Deficits due to Alzheimer's Disease or Vascular Diseases through Regulating Neuroplasticity
}

\author{
Shaozhen Ji $\mathbb{D}^{1,2,3}$ Jiayu Duan, ${ }^{1,4}$ Xiaobing Hou, ${ }^{5}$ Li Zhou, ${ }^{6}$ Weilan Qin, ${ }^{6}$ Huanmin Niu, ${ }^{5}$ \\ Shuyun Luo, ${ }^{5}$ Yunling Zhang, ${ }^{7}$ Piu Chan $\left({ }^{1},{ }^{2,3}\right.$ and Xianglan Jin $\left({ }^{1}{ }^{1}\right.$ \\ ${ }^{1}$ Department of Neurology, Dongfang Hospital, Beijing University of Chinese Medicine, Beijing 100078, China \\ ${ }^{2}$ Department of Neurobiology, Xuanwu Hospital of Capital Medical University, Beijing 100053, China \\ ${ }^{3}$ National Clinical Research Center for Geriatric Disorders, Capital Medical University, Beijing 100053, China \\ ${ }^{4}$ Beijing University of Chinese Medicine, Beijing 100029, China \\ ${ }^{5}$ Department of Neurology, Beijing First Hospital of Integrated Chinese and Western Medicine, Beijing 100039, China \\ ${ }^{6}$ Department of Acupuncture and Moxibustion, Dongfang Hospital, Beijing University of Chinese Medicine, Beijing 100078, China \\ ${ }^{7}$ Xiyuan Hospital, China Academy of Chinese Medical Sciences, Beijing 100091, China \\ Correspondence should be addressed to Piu Chan; pbchan@hotmail.com and Xianglan Jin; jxlan2001@126.com
}

Received 24 June 2020; Revised 29 November 2020; Accepted 15 December 2020; Published 12 January 2021

Academic Editor: Zhen Zheng

Copyright (c) 2021 Shaozhen Ji et al. This is an open access article distributed under the Creative Commons Attribution License, which permits unrestricted use, distribution, and reproduction in any medium, provided the original work is properly cited.

\begin{abstract}
Dementia affects millions of elderly worldwide causing remarkable costs to society, but effective treatment is still lacking. Acupuncture is one of the complementary therapies that has been applied to cognitive deficits such as Alzheimer's disease (AD) and vascular cognitive impairment (VCI), while the underlying mechanisms of its therapeutic efficiency remain elusive. Neuroplasticity is defined as the ability of the nervous system to adapt to internal and external environmental changes, which may support some data to clarify mechanisms how acupuncture improves cognitive impairments. This review summarizes the up-to-date and comprehensive information on the effectiveness of acupuncture treatment on neurogenesis and gliogenesis, synaptic plasticity, related regulatory factors, and signaling pathways, as well as brain network connectivity, to lay ground for fully elucidating the potential mechanism of acupuncture on the regulation of neuroplasticity and promoting its clinical application as a complementary therapy for AD and VCI.
\end{abstract}

\section{Introduction}

As the population ages, the prevalence of dementia is increasing worldwide with an annual incidence of nearly 10 million [1], which leads to threats and challenges to global health and wellbeing. Dementia is characterized as a syndrome with myriad and complex causes, including primary neurologic, neuropsychiatric, and medical conditions and genetic and environmental factors $[2,3]$. In the elderly, neurodegenerative dementias are most common [2], among which Alzheimer's disease $(\mathrm{AD})$ is believed to be the leading cause of dementia, and vascular cognitive impairment (VCI) is the second utmost cause $[4,5]$. Unprecedented advancements have been made in molecular neuroimaging, clinicopatho- logic correlation, and the development of novel biomarkers in recent decades. However, effective therapeutics remain limited and even absent to date $[4,5]$. Acupuncture, as one of complementary therapies for $\mathrm{AD}$ and $\mathrm{VCI}$, is gradually applied to alleviate suffering, aggressively treating contributing symptoms and improving overall quality of life [6-10]. However, the underlying mechanisms remain elusive.

Neuroplasticity refers to the capacity of the nervous system to adapt to internal and external environmental changes by reorganizing its structure, function, and connections [11-14], which occurs at various levels of the nervous system from tissue to cellular to molecular [13]. It is known that dysregulated or disrupted neuroplasticity is implicated as a pathological mechanism in $\mathrm{AD}$ [15] and VCI [16]. 
Furthermore, some treatments that stimulate or modulate neuroplasticity have been indicated as effective in improving cognition $[12,17,18]$, and might be potential therapy in cognitive impairments such as AD and VCI.

Acupuncture signals are recognized as a potent form of sensory stimulation that ascend mainly through the spinal ventrolateral funiculus to the brain [19]. The mechanisms of acupuncture-mediated neuroplasticity have recently attracted increased interest. Accordingly, acupuncture modulation over several cognition- or aging-related gene expressions [20], plasticity signaling pathways [21, 22], and brain functional connectivities [23] has been studied. Herein, we review the application of different protocols of acupuncture in animal models and humans, and their effectiveness on neuroplasticity in various sections: neurogenesis and gliogenesis, synaptic plasticity, related proteins and signaling pathways, and brain network connectivity. This review is aimed at laying the ground for elucidating the potential mechanism of acupuncture on AD and VCI to promote its clinical application as a complementary treatment.

\section{Neurogenesis and Gliogenesis}

The proliferation and differentiation of neurons and glial cells, also known as neurogenesis and gliogenesis, contribute to some neurorepair and improve brain function $[24,25]$. Many previous results demonstrated that cerebral amyloidosis in AD mouse models caused neuronal proliferation inhibition and marked gliogenesis [26-28], and that stroke could trigger striatal and cortical neurogenesis and gliogenesis in murine models [29]. Mounting evidence indicates that adult hippocampal neurogenesis is implicated in cognitive processes, and that neurogenesis deficits may impair learning and memory. In states of brain injury such as AD and VCI, compensatory neurogenesis and gliogenesis mediate a balance between initial injury processes and endogenous repair processes [24]. Regulation of neurogenesis and gliogenesis is possibly associated with improving cognitive impairment and, consequently, may be attractive therapeutic targets for $\mathrm{AD}$ and VCI.

It is known that neurogenesis in the adult mammalian brain mostly takes place in specific brain regions harboring adult neuro stem and precursor cells, such as the subgranular zone (SGZ) of the hippocampal dentate gyrus (DG) and the ventricular/subventricular zone (VZ/SVZ) of the lateral ventricles [25]. Cognitive impairment due to $\mathrm{AD}$ or ischemic injury is recognized as partly related with neuron loss, impairment of cell proliferation, and imbalance between neuron loss and proliferation in the above regions [30]. Some studies showed that both manual acupuncture (MA) and electroacupuncture (EA) could ameliorate the learning and memory deficits of AD mice models through inducing the enhancement of neuron proliferation and migration in hippocampal DG and VZ/SVZ [31-33]. And the effect of MA and EA on improving cognitive dysfunction through the proliferation and differentiation of hippocampal neuro stem cells (NSCs) was also identified in murine models for vascular dementia (VaD) [34-36]. In addition, neurogenesis could take place in other brain areas in pathological conditions, such as the cortex [37], where the promotion of neurogenesis related to EA was also detected in the transgenic mice model for $\mathrm{AD}[33]$.

VCI is recognized to be associated with pathological changes in white matter degeneration and demyelination [38]. Oligodendrocyte (OL), as one predominant cell type in white matter, mediates myelination that is an essential process for the appropriate propagation of action potentials along axons [39]. Myelination participates in the restoration of damaged white matter in the adult brain [40], which may provide potential utility for the treatment of VCI. In a mouse model of $\mathrm{VaD}$, EA was indicated to enhance the differentiation of oligodendrocyte precursor cells (OPCs) into mature OLs and ameliorate white matter damage in the corpus callosum (CC) [41]. Moreover, astrocytes also perform critical impacts on promoting neovascularization, regulating neuronal activity, and supporting synaptogenesis and neurogenesis, which may influence recovery following ischemic lesion $[39,42]$. Experimental studies have reported that acupuncture was able to influence the proliferation and differentiation of astrocytes; however, the results were discrepant. One study revealed that MA was able to inhibit astrocyte activation and proliferation in $\mathrm{VaD}$ rat models [36]. Conversely, Kim et al. found that EA stimulation could induce NSCs differentiated into astrocytes in a $\mathrm{VaD}$ mouse model [35]. These results may be caused by differential acupoints or acupuncture methods. The differential influence of the acupuncture method (i.e., MA vs. EA) on neurogenesis has been demonstrated. And one study found that MA vs. EA stimulation at the same acupoints might induce differential cell proliferation and neuroblast differentiation in healthy rats [43]. And further investigation of the compared impact of differential acupuncture methods and acupoints on gliogenesis in AD and VCI models is required.

In addition to the direct effect on endogenous neurogenesis and gliogenesis, acupuncture was able to promote the survival, proliferation, migration, and differentiation of exogenous NSCs in the hippocampal microenvironment by regulating components of the cerebral microenvironment [44] or the related cytokine levels [45] in an AD mice model. All these findings demonstrated the influence of acupuncture on endogenous and exogenous neurogenesis and gliogenesis in $\mathrm{AD}$ and VCI, which deepen our understanding of acupuncture modulating neuroplasticity. There remain some limitations and even discrepancies in these results possibly caused by acupoints or models or observation times, or even acupuncture methods (i.e., MA vs. EA). And the mechanisms underlying the impact of acupuncture on neurogenesis and gliogenesis in different states, especially molecular mechanisms, need to be investigated.

\section{Synaptic Plasticity}

Synapses, the most sensitive and plastic structures, are directly involved in the integration and transfer of information within the neuro system. Previous studies demonstrated that synapse loss and dysfunction was a key feature in $\mathrm{AD}$ [46] and VCI [47] and positively correlates with cognitive damage. Impaired dendritic structure, spine density, and 
synaptic ultrastructure of neurons have been identified in brain tissue of $\mathrm{AD}$ patients and murine models, caused by soluble amyloid beta $(\mathrm{A} \beta)$ in the hippocampus $[48,49]$. And ischemia-induced synapse reduction was also recognized to be the major pathological causes of $\mathrm{VaD}$ [50]. Synaptic plasticity, also defined as activity-dependent synaptic modifications of the strength of synaptic connections, is widely recognized to be fundamental to the formation and maintenance of learning and memory [51]. Synaptic plasticity in the neuro network, an important basis for cortical plasticity, is associated with learning and memory and sensorimotor dysfunction and recovery [51, 52]. Synaptic plasticity mainly includes modulation of the morphological structure of synapses and the synaptic strength and transmission, in which some synaptic protein markers, neurotransmitters, and receptors participate. Recently, modulation of synaptic plasticity is believed to be a promising approach for treating $\mathrm{AD}$ and VCI.

Synapse-structure parameters, such as synaptic curvatures, the width of the synaptic cleft, and the thickness of the postsynaptic density, are proposed to be important indicators that reflect synaptic morphological plasticity and greatly affect synaptic transmission [53]. Many studies revealed that MA and EA treatments had positive effects on the recovery of the learning and memory abilities not only in $\mathrm{AD}$ rat models but also in VCI, through increasing synaptic curvatures, decreasing the width of synaptic clefts, and thickening the postsynaptic densities in the hippocampus $[49,54]$. In addition, MA was able to reverse the learning and memory impairments in $\mathrm{AD}$ mice models through enhancing the conjunction among the synapses and promoting synaptic formation [20] and regeneration [55], reducing ultrastructural degradation of synapses [56], and increasing the number and length of dendrites [57] and neurite fibers [58].

Long-term potentiation (LTP) and long-term depression (LTD) are considered as two indicators and forms of synaptic transmission [59]. As a cellular model of synaptic plasticity, LTP is the long-lasting enhancement in signal transmission between two neurons after synchronous stimulation associated with memory formation and storage, reflecting an increase of synaptic strength [60]. LTD is relevant to memory integration, forgetting, and recovery of LTP production at desaturation state [61]. And converging studies supported a crucial role of LTD in some types of learning and memory and in situations where cognitive demands require a flexible response [59]. Many electrophysiological studies showed that acupuncture could apparently improve the recovery from cognitive deficits by promoting LTP and/or LTD [61-63] and preventing or restoring the impaired LTP [64-69] in $\mathrm{AD}$ or VCI rat models. In addition to LTP and LTD, EA could also ameliorate the synaptic transmission by raising the slope of excitatory postsynaptic potential (EPSP) and the amplitude of population spikes (PS) in an $\mathrm{AD}$ mouse model [70].

Synaptophysin (SYN) is a major integral membrane protein of the presynaptic vesicle, and postsynaptic density 95 (PSD-95) and growth-associated protein 43 (GAP-43) are postsynaptic markers [71]. As important protein markers of regeneration and remodeling, they are widely found in all nerve terminals and used for quantifying the number of axon terminals, reflecting the occurrence, density, and strength of synapses [49, 72]. Many previous studies reported reduced expression of SYN and PSD-95 in the hippocampus in $\mathrm{AD}$ and $\mathrm{VaD}[73,74]$. It was demonstrated that acupuncture was able to promote synapse-structure damage rehabilitation by upregulating the expression of SYN $[44,54,55]$, PSD-95 $[56,75,76]$, and GAP-43 [77] to improve the learning and memory abilities of AD and VCI murine models.

Furthermore, accumulated evidence indicates that the effect of acupuncture on modulating synaptic structure and function in $\mathrm{AD}$ and VCI is achieved by changing the releasing of the presynaptic neurotransmitter or the function of the postsynaptic receptor $[67,68,78]$. As one of the major neurotransmitters, dopamine (D) plays an essential role in modulating hippocampal LTP and memory processes [79, 80]. Ye et al. found that MA could activate D1/D5 receptors to ameliorate cognitive function and LTP impairments in $\mathrm{VaD}$ rats [67]. The central cholinergic pathway and the norepinephrine- (NE) adrenergic receptor (AR) system are known for their critical roles in learning acquisition and synaptic plasticity in the mammalian limbic system. It was demonstrated that MA not only could alleviate memoryassociated decreases in the levels of choline acetyltransferase (ChAT) and restore the expression of choline transporter 1 (CHT1) as well as vesicular acetylcholine transporter $(\mathrm{AChT})$, resulting eventually in the recovery of the entire cholinergic system circulation pathway [81], but also was able to enhance norepinephrine (NE) levels and the activation of $\beta 1$-AR in the hippocampus [68]. In addition, $\gamma$-aminobutyric acid (GABA) is one main inhibitory neurotransmitter in the central nervous system inhibiting the excessive release of glutamate (Glu). And GABA receptormediated inhibitory inputs modulate hippocampal LTP [82]. EA could elevate the excitability of granule cells by decreasing GABA from interneurons, which resulted in increasing LTP [78].

Glutamate receptors (GluRs) are the main receptors of the postsynaptic neurotransmitter area and modulate synaptic plasticity; they are divided into metabotropic GluRs and ionotropic GluRs. Among the three types of ionotropic GluRs, N-methyl-D-aspartate receptor (NMDAR) is the most widely distributed regulator of synaptic plasticity, which plays an important role in inducing and maintaining LTP and LTD closely associated with learning and memory [83]. NMDARs are comprised of NMDAR subtype 1 (NMDAR1) subunits plus at least one type of NMDAR2 subunit [84]. It was reported that EA could reduce the deficit of LTP in $\mathrm{VaD}$ rat models via reversal of NMDAR1- and transient receptor potential vanilloid subtype 1- (TRPV1-) mediated neurotoxicity [62]. NMDAR2 seems to have complex properties, and different NMDAR2 subunits confer distinct electrophysiological and pharmacological properties on the receptors and couple themselves with opposing signaling pathways and influences on the direction of synaptic plasticity [85]. Specifically, NMDAR2A activation is beneficial for neuronal regeneration and neuroprotection, while NMDAR2B induces neurotoxicity and neuronal apoptosis [85]. One study found that EA could alleviate cognitive 
dysfunction caused by ischemic injury through downregulation of NMDAR2B and upregulation of NMDAR2A [86].

The effect of EA on synaptic plasticity might be related to the parameter of stimulation. One study has found that high-frequency EA may yield a stronger protective effect on hippocampal synaptic plasticity compared with low- or medium-frequency EA in $\mathrm{AD}$ rat models [61]. Further research focusing on ascertaining the optimum acupuncture parameter is required. Moreover, besides these mechanisms described above, many synaptic-related proteins or signaling pathways were required in maintaining synaptic structural plasticity and synaptic transmission. Investigations of synaptic plasticity-related regulatory factors and signaling mechanisms have been performed in many studies, and these are going to be described in Section 4 .

\section{Neuroplasticity-Related Regulatory Factors and Signaling Pathways}

Multiple crucial steps are involved in the process of neuroplasticity, which include many layers of regulation, composed of both intrinsic and extrinsic mechanisms. For example, there are a number of coordinated cell-intrinsic programs and external signals involved in distinct stages of adult neurogenesis, including proliferation and lineage differentiation of NSCs, migration of neuroblasts, and integration of newborn neurons [87]. Given the important role of related factors and signaling pathways in neuroplasticity, ascertaining acupuncture's effect on them may be vital to understanding the mechanisms of its treatment for $\mathrm{AD}$ and VCI.

As one of the morphogens that are critical during embryonic development of the nervous system, Notch is highly conserved and serves as niche signals to regulate the proliferation of adult NSCs [88]. The regeneration of neurons from neural progenitors may be impaired due to the abnormal elevated Notch signal pathway. EA treatment suppressed neuronal apoptosis and improved cognitive impairment in $\mathrm{AD}$ rat models possibly via the downregulation of an abnormal elevated Notch signaling pathway [89]. Moreover, EA also was able to enhance hippocampal NSC proliferation in $\mathrm{VaD}$ rat models via the activation of the Notch signaling pathway [34].

In addition to the neurotransmitters described above, the survival and synaptic integration of newly born cells are subject to regulation by neurotrophic factors. As a member of the neurotrophic factor family, the BDNF protein is synthesized as pre-pro-BDNF and cleaved intracellularly into a pro-BDNF protein encompassing two domains: the prodomain and the mature BDNF domain [90]. BDNF is actually secreted in the pro- and mature form [91], which had distinct receptors and signaling cascades resulting in opposing biological functions [92-94]. The mature BDNF preferentially binds to phosphorylated tropomyosin receptor kinase $\mathrm{B}$ (Trk-B) receptors leading to cell survival and differentiation as well as hippocampal LTP, whereas pro-BDNF preferentially binds to p75 neurotrophin receptor (p75NTR) leading to apoptosis and hippocampal LTD [95]. It was observed that acupuncture could upregulate the expression of Trk-B receptors and could decrease the expression level of p75NTR in
$\mathrm{AD}$ and $\mathrm{VaD}$ murine models, influence the modulation and processing of the BDNF protein from pro-BDNF to mature BDNF [33, 96, 97], and eventually enhance the mRNA expression levels of mature BDNF [35, 45, 54, 81]. One clinical trial showed that combined scalp acupuncture and cognitive training could improve the cognitive function and BDNF levels of peripheral blood in patients with stroke during the recovery stage [98]. Other extrinsic factors such neurotrophin 3 (NT3), NT4, and NT5 also play an important role in the regulation of neuronal integration [99]. EA treatment has been reported to increase the expression of NT4/5 and their receptor, tyrosine receptor Trk-B, and promote OL regeneration in association with cognitive functional improvements in a $\mathrm{VaD}$ mice model [41]. In addition, acupuncture also could regulate intrinsic factors associated with neuronal integration. For instance, MA was demonstrated to restore the expression of cAMP-response element-binding protein (CREB) mRNA in the hippocampus of rats with cognitive impairment [81].

The typical pathological hallmarks of $\mathrm{AD}$ include extracellular $\mathrm{A} \beta$ plaques and intracellular neurofibrillary tangles (NFTs) composed of hyperphosphorylated tau proteins, both of which resulted in the loss and morphological changes of dendritic spines, directly leading to the damage of neuronal synaptic function and neuroplasticity [100]. Many studies showed that acupuncture could regulate neuroplasticity by directly reducing $\mathrm{A} \beta$ deposition $[56,101]$, and some related proteins and signaling pathways participated in this process. Glycogen synthase kinase 3 beta (GSK3 $\beta$ ) is a serine/threonine protein kinase that plays a crucial role in $\mathrm{AD}$ pathogenesis, and its hyperactivity or overexpression is increasingly shown to be closely related to $\mathrm{A} \beta$ generation, tau hyperphosphorylation, and synaptic plasticity [102]. Inhibition of GSK $3 \beta$ has been indicated to increase the number of synapses and postsynaptic density thickness, and rescue the reduction of spine density in the hippocampus of an AD model. It has been revealed that EA could promote synapse-structure damage rehabilitation by downregulating GSK3 $\beta$ to improve the learning and memory abilities of AD rat models $[49,77]$. As the downstream target of GSK $3 \beta$, the reactivation of mTOR restored the acidification of the autophagy lysosome, further promoting the autophagy clearance of pathological $\mathrm{A} \beta$ plaque load [103]. Yu et al. found that EA rescued structural and functional synaptic plasticity impairments and memory deficits in AD rat models through the inactivation of GSK3 $\beta / \mathrm{mTOR}$ signaling [21]. Moreover, $\beta$-site amyloid precursor protein cleaving enzyme 1 (BACE1) is the key protein involved in $\mathrm{A} \beta$ peptide generation. One study indicated that EA could downregulate the expression of BACE1 in one AD mouse model [64].

There are some regulated factors and signaling pathways directly involved in the modulation of LTP. Protein kinase A (PKA) is a predominantly positive modulator of LTP in the hippocampus and has been demonstrated to indispensably participate in the efficacy of hippocampus-based memory [104]. Tang et al. found that EA could upregulate PKA activation, enhance synaptic plasticity, and improve memory in an $\mathrm{AD}$ mice model [64]. The p70 ribosomal protein S6 (p70S6) kinase/ribosomal protein S6 signaling pathway has 


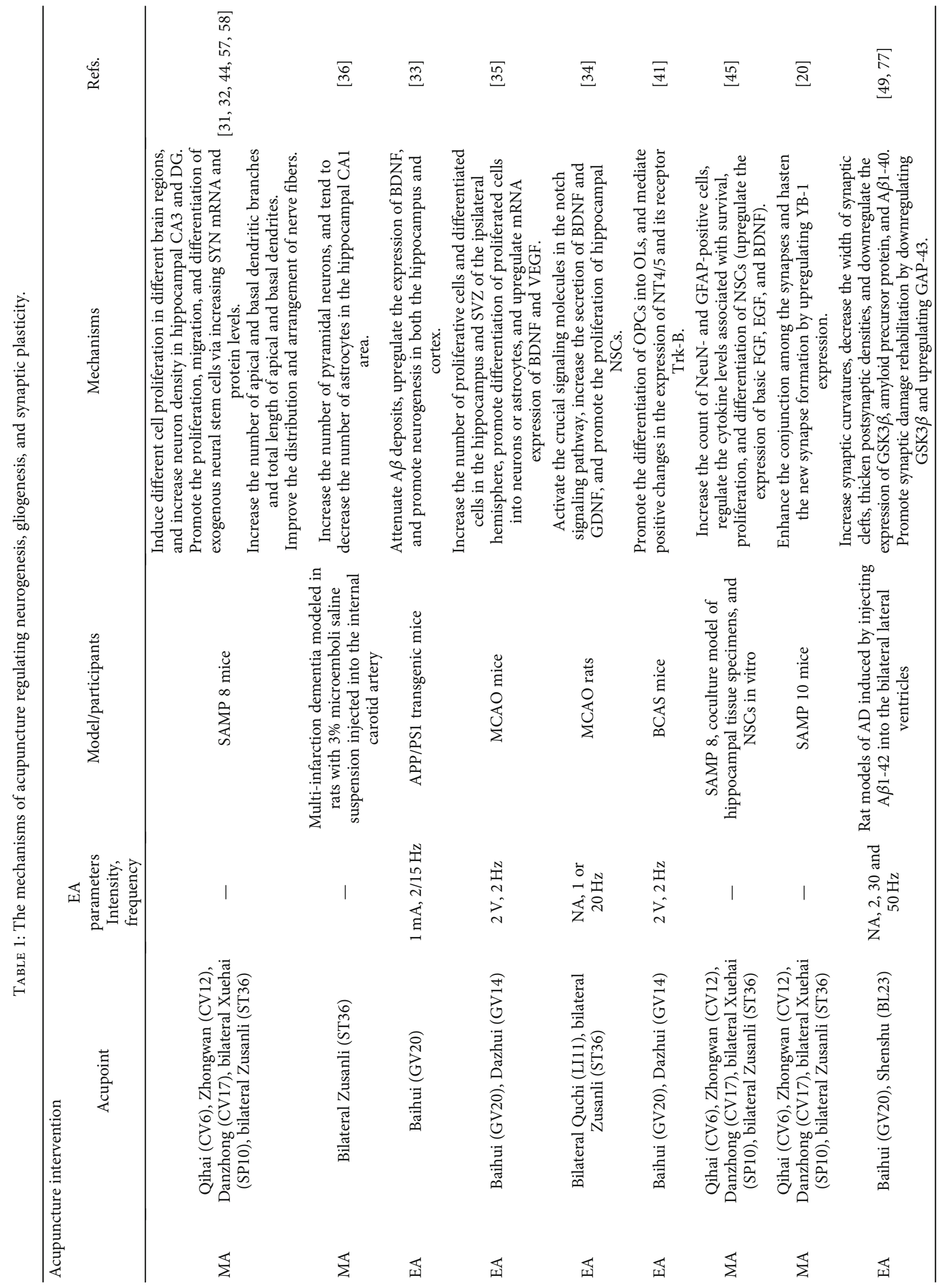




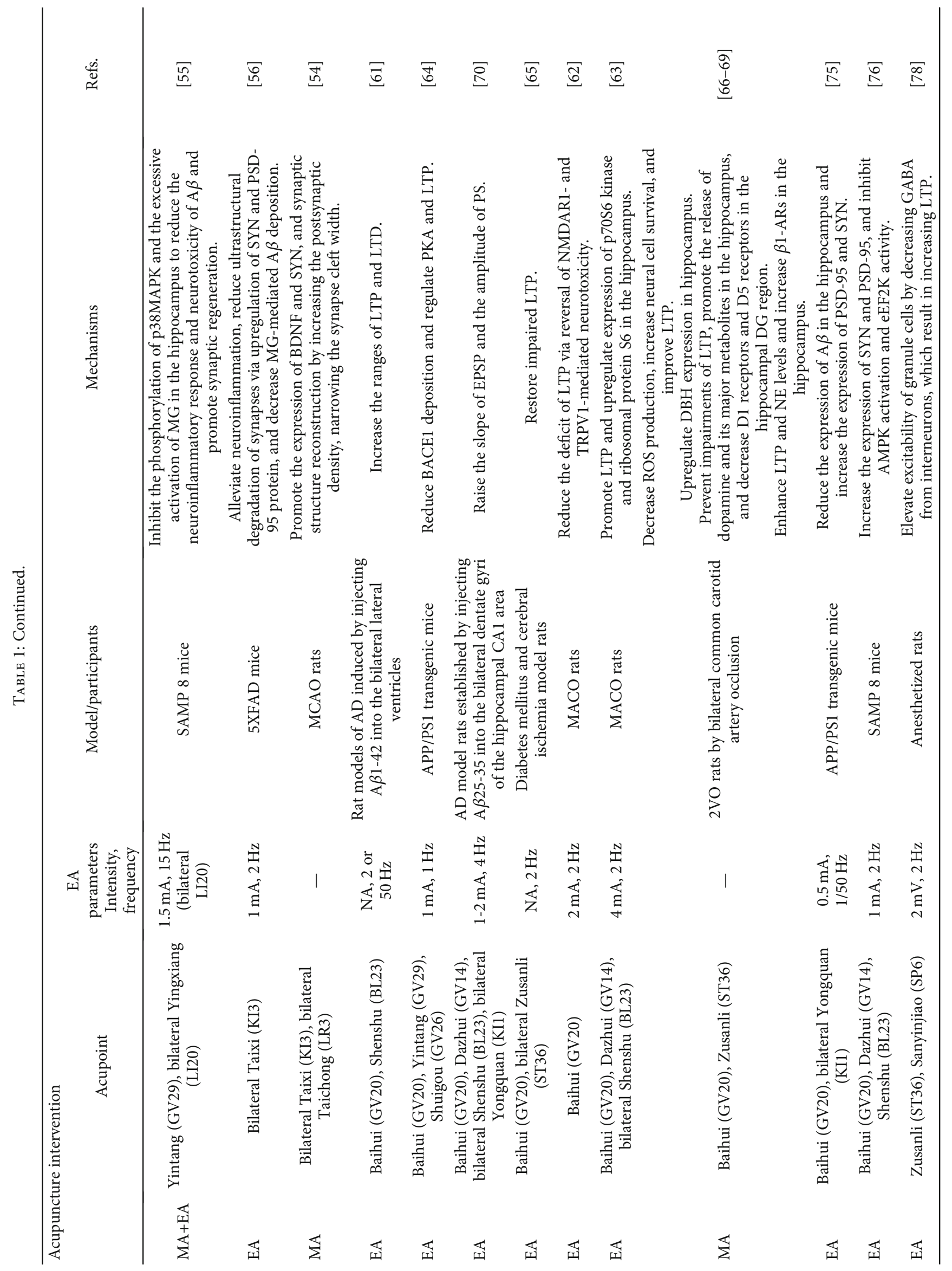




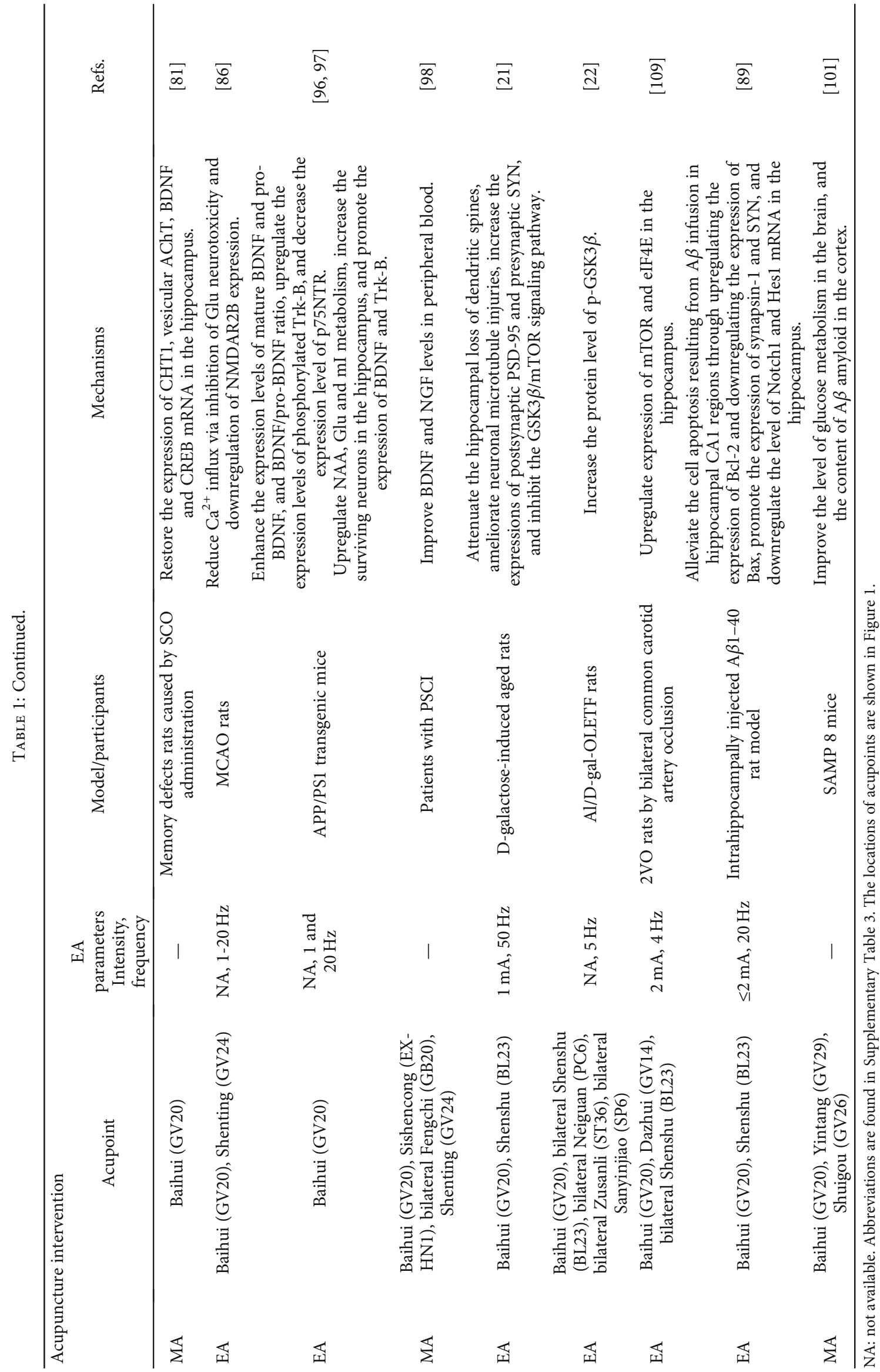



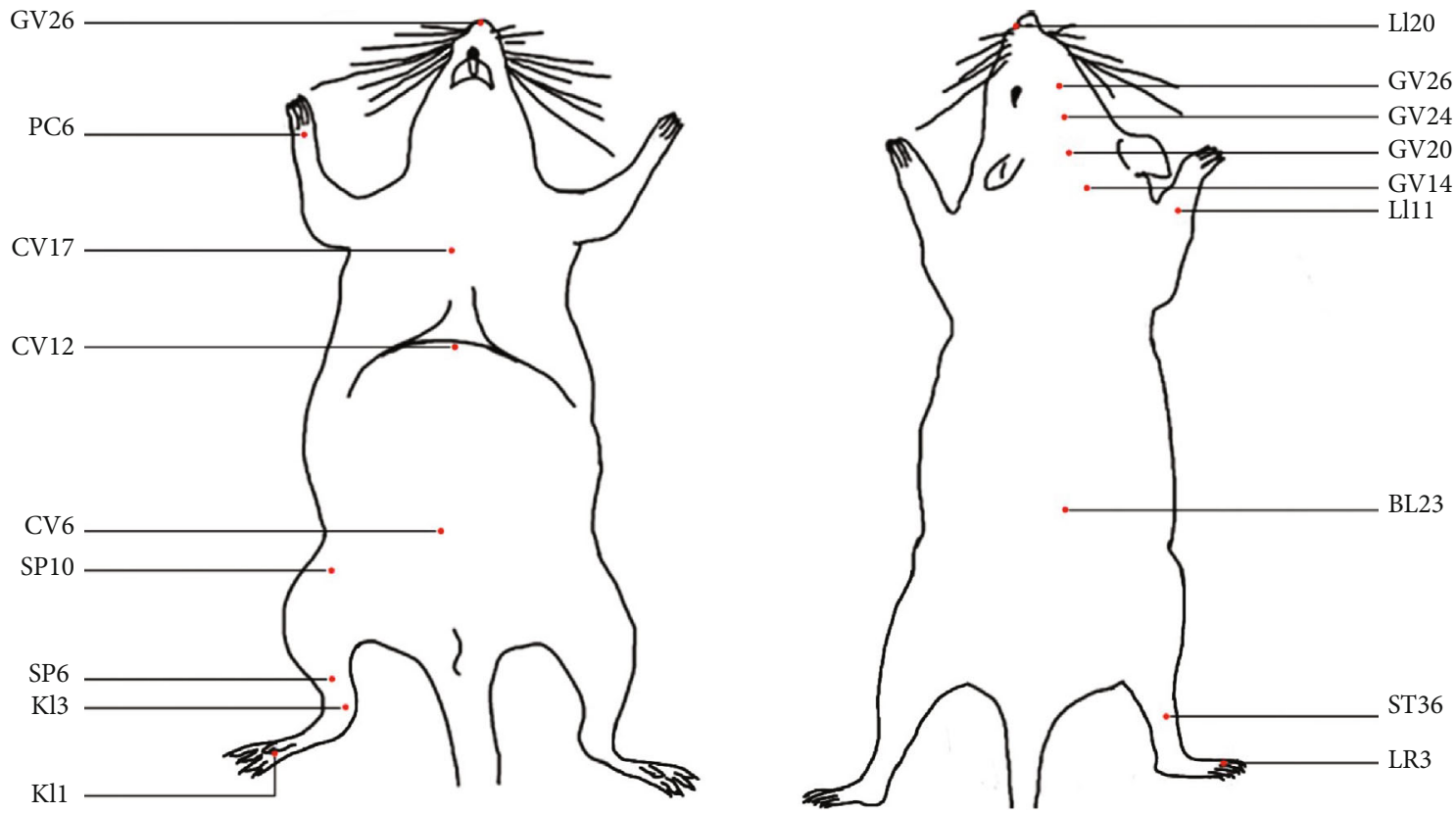

Figure 1: The locations of acupoints in mice.

been shown to promote neuronal growth and LTP $[105,106]$. One study showed reduced expression of p70S6 kinase and ribosomal protein $\mathrm{S} 6$ in the hippocampus of $\mathrm{VaD}$ rats, which suggested that the p70S6 kinase/ribosomal protein S6 pathway was involved in the pathogenesis of $\mathrm{VaD}$ [63]. EA was demonstrated to improve the learning and spatial memory abilities of $\mathrm{VaD}$ rats and facilitate LTP in the hippocampus by upregulating expression of p70S6 kinase and ribosomal protein S6 [63]. The p70S6 kinase was phosphorylated by activation of the mammalian target of rapamycin (mTOR) signal pathway, which has been shown to promote neuronal growth and LTP [107, 108]. Acupuncture stimulation has been indicated to promote neuroplasticity by regulating the mTOR signal pathway in $\mathrm{AD}$ or $\mathrm{VaD}$ rats $[21,109]$. Moreover, it was reported that MA could reverse the learning and memory impairments in an AD mouse model through upregulating eukaryotic Y-box-binding protein (YB-1) expression [20], which enhanced the conjunction among the synapses and promoted synaptic formation indirectly [110]. The eukaryotic elongation factor-2 kinase/eukaryotic elongation factor-2 (eEF2K/eEF2) pathway is also associated with synaptic plasticity and its inhibition prevents synaptic failure in $\mathrm{AD}$. One study showed that EA improved the synaptic function in $\mathrm{AD}$ by inhibiting the AMPK/eEF2K/eEF2 pathway in an AD mouse model [76].

Besides the above-related factors and signaling pathways, other mechanisms, such as oxidative stress, glucose metabolism, and inflammatory responses, were considered to play a key role in acupuncture treating $\mathrm{AD}$ or $\mathrm{VCI}$ and modulating neuroplasticity (Table 1 and Figure 1). These molecular mechanisms support acupuncture as a potentially promising therapy for the treatment of cognitive dysfunction in patients with VD or VCI.

\section{Brain Network Connectivity}

Some previous neuroimaging researches have revealed neuropathological changes and/or structural-functional reorganization in $\mathrm{AD}$ and VCI resulting in altered connectivity patterns in brain networks [14,111-113]. For example, some rapidly and reversibly increased or decreased strengths of brain network connections, also known as altered recruitments of functional connections normally devoted to performing a given task or the recruitment of additional network connections that are not typically activated by healthy people. And the alteration of network connectivity is a form of neuroplasticity that could indicate compensatory mechanisms engaged to maintain a normal level of cognitive function or promote the recovery from cognitive dysfunction due to the primary white matter lesions and neuronal loss $[14,114,115]$.

Many neuroimaging studies showed that acupuncture could induce neuroplastic reorganization of brain functional networks in $\mathrm{AD}$ or mild cognitive impairment (MCI), the prophase state of AD [116] (Table 2 and Figure 2). There were several regions showing increased or decreased activities in $\mathrm{MCI}$ and $\mathrm{AD}$ patients after short-term MA or EA stimulation, including cognitive-related areas, visual-related areas, sensorimotor-related areas, emotion-related areas, the basal ganglia, and the cerebellum [23, 113, 117-123]. However, there remains a lack of correlation between the changes in cognitive function and alteration in functional connectivity. In two other long-term studies, MCI patients exhibited improvement of cognitive performance after MA, as well as extensive activation and deactivation in brain networks $[123,124]$. And functional connectivity strength in some regions was negatively correlated with the changes in 


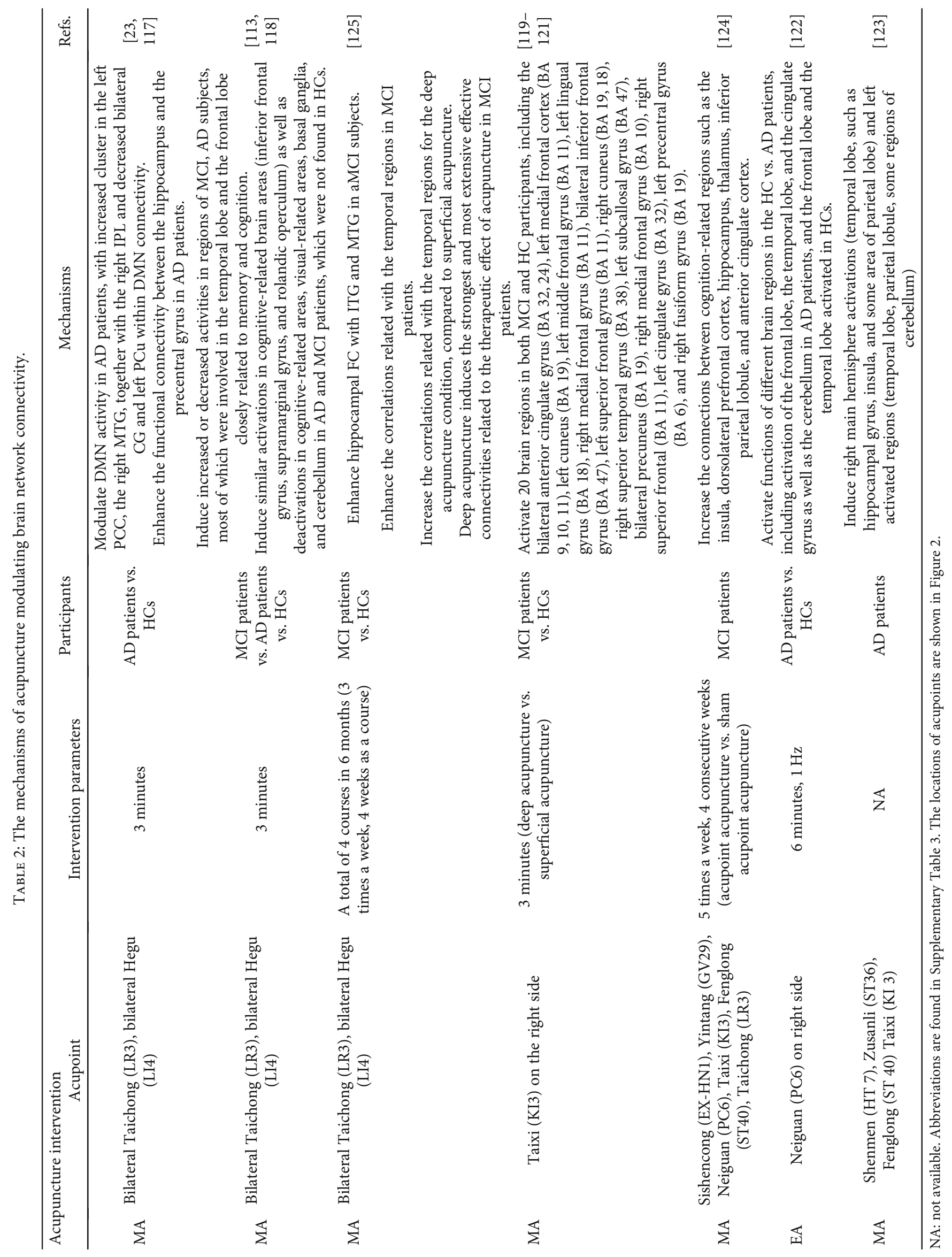



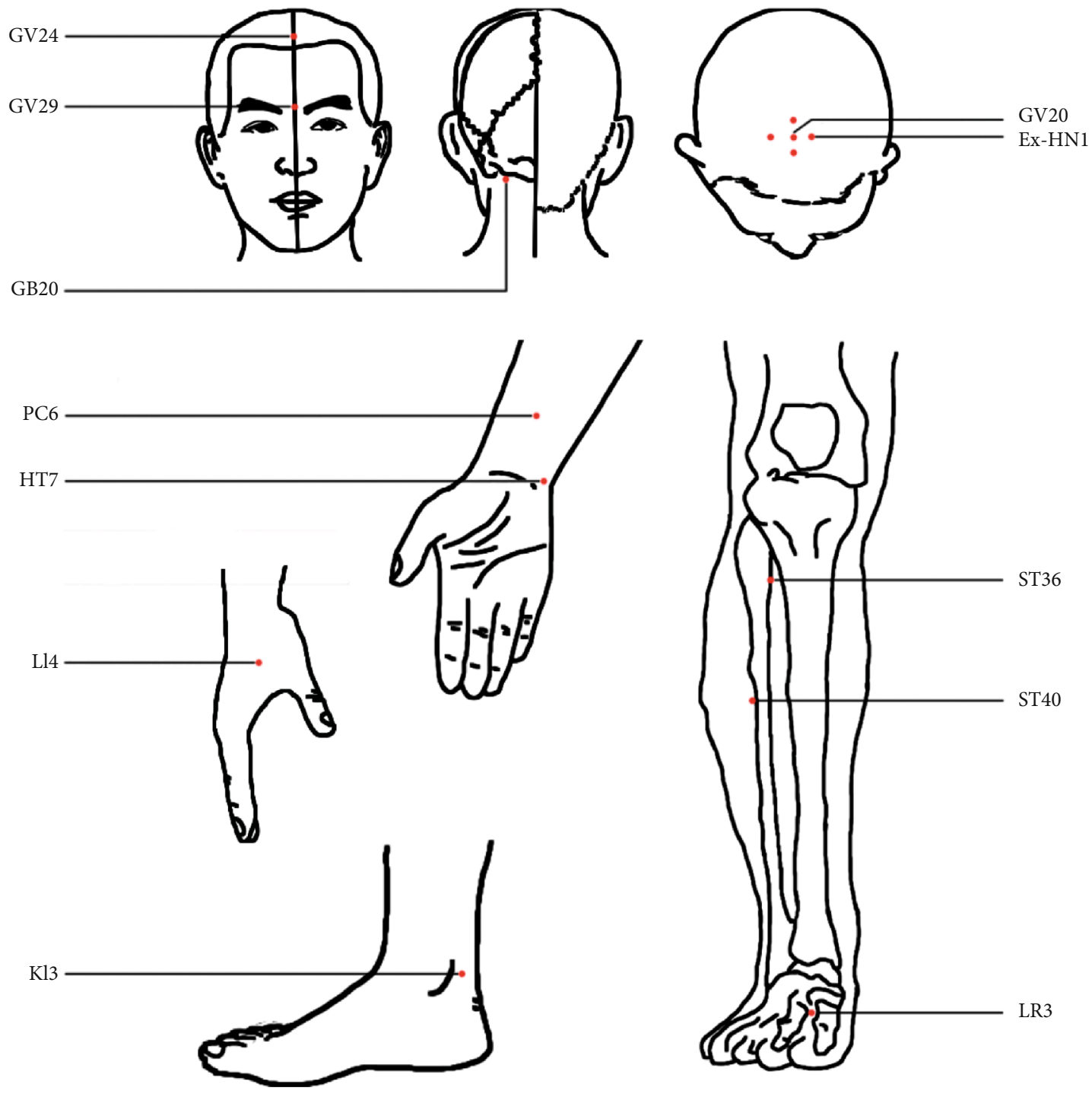

FIgUre 2: The locations of acupoints in humans.

memory scores [125], which offered evidence in support of compensatory mechanisms triggered to overcome cognitive deficits in MCI. These findings might provide a deep understanding of acupuncture's therapeutic effect in AD.

Acupuncture's influence on brain network connectivity might be correlated to acupoints, depth of stimulation, and frequency of EA stimulation in $\mathrm{AD}$ and MCI. The synergistic effects of different single acupoints or combined acupoints could activate different brain areas and impact the therapeutic effects of acupuncture [116]. And deep stimulation at appropriate acupoints could perform stronger or more extensive effective connectivity related to the therapeutic effect compared with superficial stimulation [119, 121, 126]. Furthermore, high-frequency EA may induce more specific targeted brain response or strengthen the functional connectivity of brain networks associated with memory and cognition. Thus, the impact of acupoint specificity, needling depth specificity, and EA parameter specificity on brain network connectivity in future neuroimaging studies also needs to be elucidated. Since few fMRI imaging studies have been reported regarding acupuncture in patients with VCI, the effect of acupuncture on neuroplastic reorganization of brain functional networks in VCI is still to be established.

\section{Discussion}

In addition to directly attenuating the deposition, neuroinflammatory response, and neurotoxicity of $\mathrm{A} \beta$ [127] and increasing cerebral blood flow [128], acupuncture also could improve cognitive abilities through regulation of neuroplasticity (Figure 3 ). The improvement of the cellular/molecular microenvironment and recruitment of unaffected or additional brain networks might play important roles in this process. For example, the modulation of the neurotransmitter system involved in the improvement of the cellular/molecular microenvironment may be another candidate potential mechanism through which acupuncture could regulate neuroplasticity [44]. Moreover, it has been demonstrated that other methods in popular practice could increase cognitive reserve and resilience by regulating neuroplasticity, e.g., 


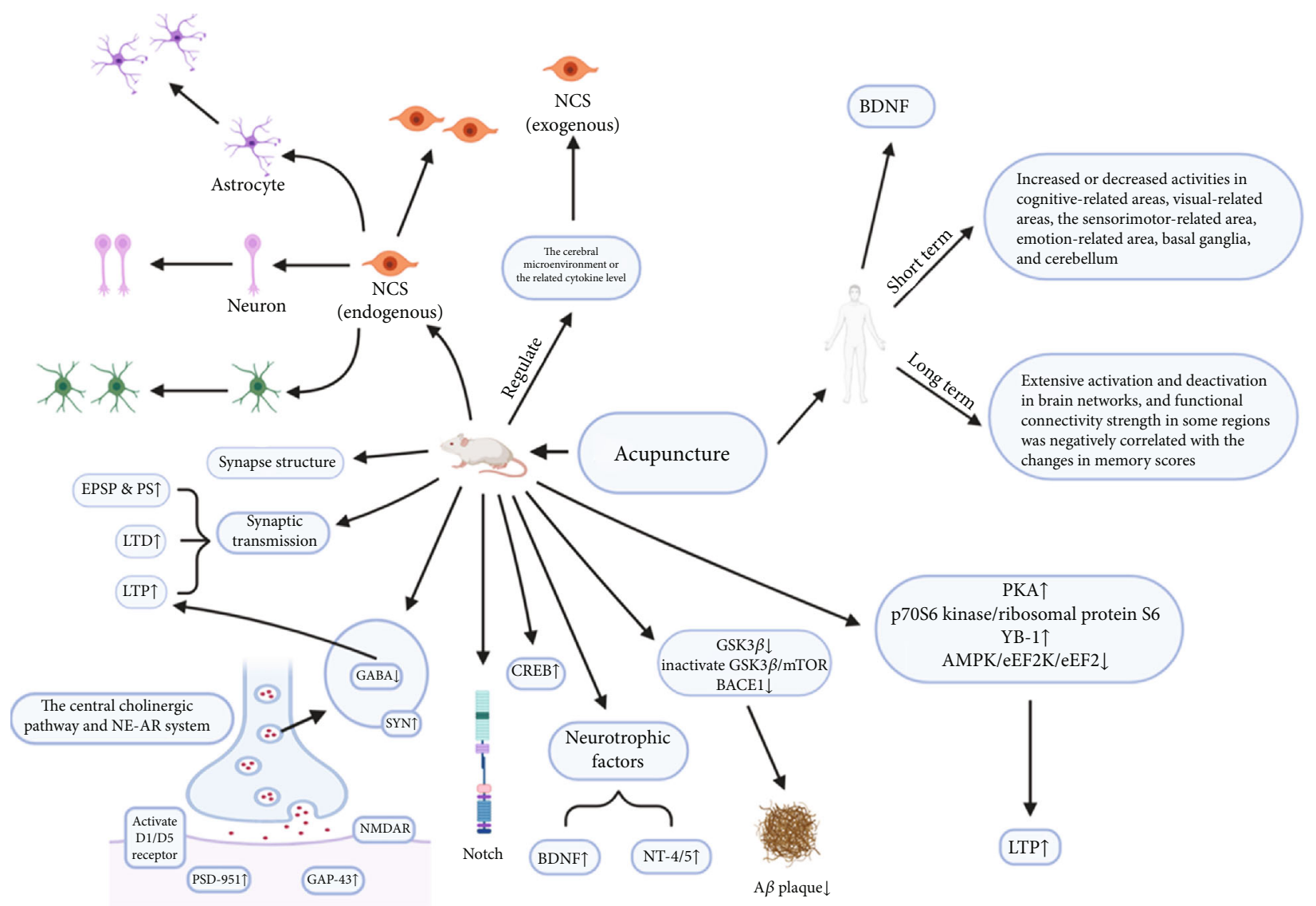

FIgURE 3: Mechanisms involved in acupuncture regulating neuroplasticity to improve cognitive function.

physical exercises, stimulating psychosocial experiences, meditation, mind games/puzzles, or dietary changes. It will be interesting to investigate whether acupuncture could increase cognitive reserve and resilience in the elderly. And the results would greatly expand the clinical application of acupuncture. Furthermore, identification of differential impacts of manipulation on brain networks may contribute to understanding the mechanisms of acupuncture in neuroplasticity. The comparison between acupuncture and sham/placebo acupuncture occurred in few clinical studies [124], which indicated increased connections between cognitionrelated regions by acupuncture not sham/placebo acupuncture. In the further researches, diffusion tensor imaging (DTI) of white matter microstructure adjacent to the primary somatosensory cortex and magnetic resonance spectroscopy (MRS) would be used to explore potential differential mechanisms of manipulation.

There are still some inevitable limitations in this review. First of all, because of differences in the quality of included animal studies, such as sample size calculations, experimental animals and procedures, housing and husbandry conditions, intervention, and assessment of experimental outcomes, the heterogeneities cannot be totally avoided (Supplementary Table 1). Second, it is well known that the efficacy of acupuncture stimulation was partly defined by the characteristic sensation "de qi" clinically (a composite of sensations including soreness, numbness, distention, heaviness, and other sensations) [129]. The efficacy of interventions could not be estimated in animal studies. Third, there are differential influences on neuroplasticity due to acupuncture manipulation. For instance, experimental outcomes may be differently attributed to intervention performed by the acupuncture method (i.e., MA vs. EA) and acupoints $[35,36]$. Since the number of studies was small, some pathways affected by the manipulation of acupuncture were not discussed, for instance, synaptophysin expression, modulation of neurotransmitter, and neuroplastic reorganization of brain functional networks (Supplementary Table 2).

\section{Conclusion}

A growing number of contemporary studies have gradually validated acupuncture's traditional uses in treating $\mathrm{AD}$ and VCI. In view of acupuncture's therapeutic efficiency and regulation of neuroplasticity, it may be beneficial to develop acupuncture as a potentially promising therapy for AD and VCI. However, the exact mechanisms underlying acupuncture's influence on neuroplasticity is still unknown. In addition, identification of differential impacts of acupoint specificity, acupuncture method specificity, depth specificity, cognitive state specificity, and EA parameter specificity on neuroplasticity may contribute to understanding the mechanisms of acupuncture in AD and VCI. These may be important future challenges in standardized clinical applications. 


\section{Data Availability}

Previously reported data were used to support this study and are cited at relevant places within the text as references.

\section{Conflicts of Interest}

The authors declare that they have no conflicts of interest.

\section{Authors' Contributions}

Shaozhen Ji, Piu Chan, and Xianglan Jin contributed in study concept and design. Shaozhen Ji and Jiayu Duan contributed in literature search. Shaozhen Ji contributed in drafting the manuscript. Shaozhen Ji, Jiayu Duan, Xiaobing Hou, Li Zhou, Weilan Qin, Piu Chan, and Xianglan Jin contributed in critical revision of the manuscript for important intellectual content. Piu Chan and Xianglan Jin contributed in obtaining funding. Xiaobing Hou, Li Zhou, Weilan Qin, Huanmin Niu, Shuyun Luo, Yunling Zhang, Piu Chan, and Xianglan Jin contributed in administrative, technical, or material support. Yunling Zhang, Piu Chan, and Xianglan Jin contributed in study supervision. Shaozhen Ji and Jiayu Duan contributed equally to this work.

\section{Acknowledgments}

The study was supported by grants 2018YFC1312001 and 2017YFC0840105 from the National Key R\&D Program of China; grant SML20150803 from the Beijing Municipal Administration of Hospitals' Mission Plan; grant Z171100000117013 from the Beijing Municipal Science \& Technology Commission; and grants 2020-JYB-ZDGG-124 and 2021-JYB-XJSJJ071 from the Fundamental Research Funds for the Central Universities of China. Xianglan Jin is supported by the Talent Cultivation Plan for Inheritance of Clinical Characteristic Technology of Chinese Medicine.

\section{Supplementary Materials}

We describe characteristics of included animal studies in Supplementary Table 1 referring to the new ARRIVE guidelines, as well as characteristics of included human studies in Supplementary Table 2. Some abbreviations included in this review are listed in Supplementary Table 3. (Supplementary Materials)

\section{References}

[1] H. Zhang, L. Hardie, A. O. Bawajeeh, and J. Cade, "Meat consumption, cognitive function and disorders: a systematic review with narrative synthesis and meta-analysis," Nutrients, vol. 12, no. 5, p. 1528, 2020.

[2] S. A. Gale, D. Acar, and K. R. Daffner, "Dementia," The American Journal of Medicine, vol. 131, no. 10, pp. 1161$1169,2018$.

[3] L. Fratiglioni, A. Marseglia, and S. Dekhtyar, "Ageing without dementia: can stimulating psychosocial and lifestyle experiences make a difference?," The Lancet Neurology, vol. 19, no. 6 , pp. 533-543, 2020.
[4] C. Iadecola, M. Duering, V. Hachinski et al., "Vascular Cognitive Impairment and Dementia:," Journal of the American College of Cardiology, vol. 73, no. 25, pp. 3326-3344, 2019.

[5] M. Revi, "Alzheimer's disease therapeutic approaches," Advances in Experimental Medicine and Biology, vol. 1195, pp. 105-116, 2020.

[6] C. Y. Hung, X. Y. Wu, V. C. Chung, E. C. Tang, J. C. Wu, and A. Y. Lau, "Overview of systematic reviews with metaanalyses on acupuncture in post- stroke cognitive impairment and depression management," Integrative Medicine Research, vol. 8, no. 3, pp. 145-159, 2019.

[7] Z. B. Liu, W. M. Niu, X. H. Yang, and X. M. Niu, "Clinical investigation on electroacupuncture treatment of vascular dementia with "Xiusanzhen", Zhen Ci Yan Jiu, vol. 33, no. 2, pp. 131-134, 2008.

[8] S. Wang, H. Yang, J. Zhang et al., "Efficacy and safety assessment of acupuncture and nimodipine to treat mild cognitive impairment after cerebral infarction: a randomized controlled trial," BMC Complementary and Alternative Medicine, vol. 16, no. 1, p. 361, 2016.

[9] Y. Y. Wang, S. F. Yu, H. Y. Xue, Y. Li, C. Zhao, and Y. H. Jin, "Effectiveness and safety of acupuncture for the treatment of Alzheimer's disease: a systematic review and meta-analysis," Frontiers in Aging Neuroscience, vol. 12, p. 98, 2020.

[10] J. W. Yang, G. X. Shi, S. Zhang et al., "Effectiveness of acupuncture for vascular cognitive impairment no dementia: a randomized controlled trial," Clinical Rehabilitation, vol. 33, no. 4, pp. 642-652, 2019.

[11] J. D. Sweatt, "Neural plasticity and behavior-sixty years of conceptual advances," Journal of Neurochemistry, vol. 139, Suppl 2, pp. 179-199, 2016.

[12] S. C. Cramer, M. Sur, B. H. Dobkin et al., "Harnessing neuroplasticity for clinical applications," Brain, vol. 134, no. 6, pp. 1591-1609, 2011.

[13] R. von Bernhardi, L. E. Bernhardi, and J. Eugenin, "What is neural plasticity?," Advances in Experimental Medicine and Biology, vol. 1015, pp. 1-15, 2017.

[14] A. F. Arnsten, C. D. Paspalas, N. J. Gamo, Y. Yang, and M. Wang, "Dynamic network connectivity: a new form of neuroplasticity," Trends in Cognitive Sciences, vol. 14, no. 8, pp. 365-375, 2010.

[15] J. Jackson, E. Jambrina, J. Li et al., "Targeting the synapse in Alzheimer's disease," Frontiers in Neuroscience, vol. 13, p. 735, 2019.

[16] B. Liu, J. Liu, J. Zhang, W. Mao, and S. Li, "Effects of autophagy on synaptic-plasticity-related protein expression in the hippocampus CA1 of a rat model of vascular dementia," Neuroscience Letters, vol. 707, article 134312, 2019.

[17] E. Dayan and L. G. Cohen, "Neuroplasticity subserving motor skill learning," Neuron, vol. 72, no. 3, pp. 443-454, 2011.

[18] C. Cavaleiro, J. Martins, J. Goncalves, and M. Castelo-Branco, "Memory and cognition-related neuroplasticity enhancement by transcranial direct current stimulation in rodents: a systematic review," Neural Plasticity, vol. 2020, Article ID 4795267, 23 pages, 2020.

[19] Z. Q. Zhao, "Neural mechanism underlying acupuncture analgesia," Progress in Neurobiology, vol. 85, no. 4, pp. 355375, 2008.

[20] X. Ding, J. Yu, T. Yu, Y. Fu, and J. Han, “Acupuncture regulates the aging-related changes in gene profile expression of 
the hippocampus in senescence-accelerated mouse (SAMP10)," Neuroscience Letters, vol. 399, no. 1-2, pp. 11$16,2006$.

[21] C. C. Yu, J. Wang, S. S. Ye et al., "Preventive Electroacupuncture Ameliorates D-Galactose-Induced Alzheimer's DiseaseLike Pathology and Memory Deficits Probably via Inhibition of GSK3 $\beta / \mathrm{mTOR}$ Signaling Pathway," Evidence-based Complementary and Alternative Medicine, vol. 2020, Article ID 1428752, 12 pages, 2020.

[22] X. Huang, K. Huang, Z. Li et al., "Electroacupuncture improves cognitive deficits and insulin resistance in an OLETF rat model of Al/D-gal induced aging model via the PI3K/Akt signaling pathway," Brain Research, vol. 1740, article $146834,2020$.

[23] P. Liang, Z. Wang, T. Qian, and K. Li, "Acupuncture stimulation of Taichong (Liv3) and Hegu (LI4) modulates the default mode network activity in Alzheimer's disease," American Journal of Alzheimer's Disease and Other Dementias, vol. 29, no. 8, pp. 739-748, 2014.

[24] J. Frisen, "Neurogenesis and gliogenesis in nervous system plasticity and repair," Annual Review of Cell and Developmental Biology, vol. 32, no. 1, pp. 127-141, 2016.

[25] C. Zhao, W. Deng, and F. H. Gage, "Mechanisms and functional implications of adult neurogenesis," Cell, vol. 132, no. 4, pp. 645-660, 2008.

[26] D. Baglietto-Vargas, E. Sánchez-Mejias, V. Navarro et al., "Dual roles of $A \beta$ in proliferative processes in an amyloidogenic model of Alzheimer's disease," Scientific Reports, vol. 7, no. 1, article 10085, 2017.

[27] L. Bondolfi, M. Calhoun, F. Ermini et al., "Amyloid-associated neuron loss and gliogenesis in the neocortex of amyloid precursor protein transgenic mice," The Journal of Neuroscience, vol. 22, no. 2, pp. 515-522, 2002.

[28] L. Verret, J. L. Jankowsky, G. M. Xu, D. R. Borchelt, and C. Rampon, "Alzheimer's-type amyloidosis in transgenic mice impairs survival of newborn neurons derived from adult hippocampal neurogenesis," The Journal of Neuroscience, vol. 27, no. 25, pp. 6771-6780, 2007.

[29] O. Lindvall and Z. Kokaia, "Neurogenesis following stroke affecting the adult brain," Cold Spring Harbor Perspectives in Biology, vol. 7, no. 11, 2015.

[30] G. Li, H. Cheng, X. Zhang et al., "Hippocampal neuron loss is correlated with cognitive deficits in SAMP8 mice," Neurological Sciences, vol. 34, no. 6, pp. 963-969, 2013.

[31] G. Li, X. Zhang, H. Cheng et al., "Acupuncture improves cognitive deficits and increases neuron density of the hippocampus in middle-aged SAMP8 mice," Acupuncture in Medicine, vol. 30, no. 4, pp. 339-345, 2012.

[32] H. Cheng, J. Yu, Z. Jiang et al., "Acupuncture improves cognitive deficits and regulates the brain cell proliferation of SAMP8 mice," Neuroscience Letters, vol. 432, no. 2, pp. 111-116, 2008.

[33] X. Li, F. Guo, Q. Zhang et al., "Electroacupuncture decreases cognitive impairment and promotes neurogenesis in the APP/PS1 transgenic mice," BMC Complementary and Alternative Medicine, vol. 14, no. 1, p. 37, 2014.

[34] J. Tao, B. Chen, Y. Gao et al., "Electroacupuncture enhances hippocampal NSCs proliferation in cerebral ischemiareperfusion injured rats via activation of notch signaling pathway," The International Journal of Neuroscience, vol. 124, no. 3, pp. 204-212, 2013.
[35] Y. R. Kim, H. N. Kim, S. M. Ahn, Y. H. Choi, H. K. Shin, and B. T. Choi, "Electroacupuncture promotes post-stroke functional recovery via enhancing endogenous neurogenesis in mouse focal cerebral ischemia," PLoS One, vol. 9, no. 2, article e90000, 2014.

[36] F. Li, C. Q. Yan, L. T. Lin et al., “Acupuncture attenuates cognitive deficits and increases pyramidal neuron number in hippocampal CA1 area of vascular dementia rats," $B M C$ Complementary and Alternative Medicine, vol. 15, no. 1, p. 133, 2015.

[37] R. Lin and L. Iacovitti, "Classic and novel stem cell niches in brain homeostasis and repair," Brain Research, vol. 1628, pp. 327-342, 2015.

[38] P. B. Gorelick, A. Scuteri, S. E. Black et al., "Vascular contributions to cognitive impairment and dementia: a statement for healthcare professionals from the American Heart Association/American Stroke Association," Stroke, vol. 42, no. 9, pp. 2672-2713, 2011.

[39] A. M. Ibrahim, F. H. Pottoo, E. S. Dahiya, F. A. Khan, and J. S. Kumar, "Neuron-glia interactions: molecular basis of Alzheimer's disease and applications of neuroproteomics," The European Journal of Neuroscience, vol. 52, no. 2, pp. 29312943, 2020.

[40] R. J. Franklin and C. ffrench-Constant, "Remyelination in the CNS: from biology to therapy," Nature Reviews Neuroscience, vol. 9, no. 11, pp. 839-855, 2008.

[41] S. M. Ahn, Y. R. Kim, H. N. Kim, Y. I. Shin, H. K. Shin, and B. T. Choi, "Electroacupuncture ameliorates memory impairments by enhancing oligodendrocyte regeneration in a mouse model of prolonged cerebral hypoperfusion," Scientific Reports, vol. 6, no. 1, article 28646, 2016.

[42] M. Santello, N. Toni, and A. Volterra, "Astrocyte function from information processing to cognition and cognitive impairment," Nature Neuroscience, vol. 22, no. 2, pp. 154$166,2019$.

[43] I. K. Hwang, J. Y. Chung, D. Y. Yoo et al., "Comparing the effects of acupuncture and electroacupuncture at Zusanli and Baihui on cell proliferation and neuroblast differentiation in the rat hippocampus," The Journal of Veterinary Medical Science, vol. 72, no. 3, pp. 279-284, 2010.

[44] C. L. Zhou, L. Zhao, H. Y. Shi et al., "Combined acupuncture and HuangDiSan treatment affects behavior and synaptophysin levels in the hippocampus of senescence-accelerated mouse prone 8 after neural stem cell transplantation," Neural Regeneration Research, vol. 13, no. 3, pp. 541-548, 2018.

[45] L. Zhao, C. Zhou, L. Li et al., "Acupuncture improves cerebral microenvironment in mice with Alzheimer's disease treated with hippocampal neural stem cells," Molecular Neurobiology, vol. 54, no. 7, pp. 5120-5130, 2017.

[46] C. R. Jack Jr., D. S. Knopman, W. J. Jagust et al., "Hypothetical model of dynamic biomarkers of the Alzheimer's pathological cascade," Lancet Neurology, vol. 9, no. 1, pp. 119-128, 2010.

[47] F. Wang, Y. Cao, L. Ma, H. Pei, W. D. Rausch, and H. Li, "Dysfunction of cerebrovascular endothelial cells: prelude to vascular dementia," Frontiers in Aging Neuroscience, vol. 10, p. $376,2018$.

[48] E. Falke, J. Nissanov, T. W. Mitchell, D. A. Bennett, J. Q. Trojanowski, and S. E. Arnold, "Subicular dendritic arborization in Alzheimer's disease correlates with neurofibrillary tangle density," The American Journal of Pathology, vol. 163, no. 4, pp. 1615-1621, 2003. 
[49] C. C. Yu, Y. Wang, F. Shen et al., "High-frequency $(50 \mathrm{~Hz})$ electroacupuncture ameliorates cognitive impairment in rats with amyloid beta 1-42-induced Alzheimer's disease," Neural Regeneration Research, vol. 13, no. 10, pp. 1833-1841, 2018.

[50] H. Y. Yang, Y. Liu, J. C. Xie, N. N. Liu, and X. Tian, "Effects of repetitive transcranial magnetic stimulation on synaptic plasticity and apoptosis in vascular dementia rats," Behavioural Brain Research, vol. 281, pp. 149-155, 2015.

[51] S. A. Buffington, W. Huang, and M. Costa-Mattioli, "Translational control in synaptic plasticity and cognitive dysfunction," Annual Review of Neuroscience, vol. 37, no. 1, pp. 1738, 2014.

[52] G. Neves, S. F. Cooke, and T. V. Bliss, "Synaptic plasticity, memory and the hippocampus: a neural network approach to causality," Nature Reviews Neuroscience, vol. 9, no. 1, pp. 65-75, 2008.

[53] J. N. Bourne and K. M. Harris, "Balancing structure and function at hippocampal dendritic spines," Annual Review of Neuroscience, vol. 31, no. 1, pp. 47-67, 2008.

[54] W. G. Xia, C. J. Zheng, X. Zhang, and J. Wang, "Effects of "nourishing liver and kidney" acupuncture therapy on expression of brain derived neurotrophic factor and synaptophysin after cerebral ischemia reperfusion in rats," Journal of Huazhong University of Science and Technology. Medical Sciences, vol. 37, no. 2, pp. 271-278, 2017.

[55] Y. Wang, Q. Wang, B. Ren et al., ““'Olfactory three-needle” enhances spatial learning and memory ability in SAMP8 mice," Behavioural Neurology, vol. 2020, Article ID 2893289, 11 pages, 2020.

[56] M. Cai, J. H. Lee, and E. J. Yang, "Electroacupuncture attenuates cognition impairment via anti-neuroinflammation in an Alzheimer's disease animal model," Journal of Neuroinflammation, vol. 16, no. 1, p. 264, 2019.

[57] B. H. Kan, J. C. Yu, L. Zhao et al., "Acupuncture improves dendritic structure and spatial learning and memory ability of Alzheimer's disease mice," Neural Regeneration Research, vol. 13, no. 8, pp. 1390-1395, 2018.

[58] T. Liu, X. Z. Zhang, J. X. Han, and K. Nie, "Using bioinformatics tools to explore cellular biological mechanisms of "triple energizer acupuncture method" in treating senile dementia," Zhen Ci Yan Jiu, vol. 44, pp. 424-429, 2019.

[59] G. L. Collingridge, S. Peineau, J. G. Howland, and Y. T. Wang, "Long-term depression in the CNS," Nature Reviews. Neuroscience, vol. 11, no. 7, pp. 459-473, 2010.

[60] E. N. van den Broeke, C. M. van Rijn, J. A. Biurrun Manresa, O. K. Andersen, L. Arendt-Nielsen, and O. H. Wilder-Smith, "Neurophysiological correlates of nociceptive heterosynaptic long-term potentiation in humans," Journal of Neurophysiology, vol. 103, no. 4, pp. 2107-2113, 2010.

[61] L.-h. Kong, W. Li, H. Wang et al., "High-frequency electroacupuncture evidently reinforces hippocampal synaptic transmission in Alzheimer's disease rats," Neural Regeneration Research, vol. 11, no. 5, pp. 801-806, 2016.

[62] Y. W. Lin and C. L. Hsieh, "Electroacupuncture at Baihui acupoint (GV20) reverses behavior deficit and long-term potentiation through $\mathrm{N}$-methyl-D-aspartate and transient receptor potential vanilloid subtype 1 receptors in middle cerebral artery occlusion rats," Journal of Integrative Neuroscience, vol. 9, no. 3, pp. 269-282, 2010.

[63] Y. Zhu, X. Wang, X. Ye, C. Gao, and W. Wang, "Effects of electroacupuncture on the expression of p70 ribosomal pro- tein S6 kinase and ribosomal protein S6 in the hippocampus of rats with vascular dementia," Neural Regeneration Research, vol. 7, no. 3, pp. 207-211, 2012.

[64] Y. Tang, S. Shao, Y. Guo et al., "Electroacupuncture mitigates hippocampal cognitive impairments by reducing BACE1 deposition and activating PKA in APP/PS1 double transgenic mice," Neural Plasticity, vol. 2019, Article ID 2823679, 12 pages, 2019.

[65] X. H. Jing, S. L. Chen, H. Shi, H. Cai, and Z. G. Jin, "Electroacupuncture restores learning and memory impairment induced by both diabetes mellitus and cerebral ischemia in rats," Neuroscience Letters, vol. 443, no. 3, pp. 193-198, 2008.

[66] J. W. Yang, X. R. Wang, M. Zhang et al., "Acupuncture as a multifunctional neuroprotective therapy ameliorates cognitive impairment in a rat model of vascular dementia: a quantitative iTRAQ proteomics study," CNS Neuroscience \& Therapeutics, vol. 24, no. 12, pp. 1264-1274, 2018.

[67] Y. Ye, H. Li, J. W. Yang et al., “Acupuncture attenuated vascular dementia-induced hippocampal long-term potentiation impairments via activation of D1/D5 receptors," Stroke, vol. 48, no. 4, pp. 1044-1051, 2017.

[68] L. Y. Xiao, X. R. Wang, J. W. Yang et al., “Acupuncture prevents the impairment of hippocampal LTP through $\beta 1$-AR in vascular dementia rats," Molecular Neurobiology, vol. 55, no. 10, pp. 7677-7690, 2018.

[69] L.-Y. Xiao, J.-W. Yang, X.-R. Wang et al., “Acupuncture Rescues Cognitive Impairment and Upregulates DopamineHydroxylase Expression in Chronic Cerebral Hypoperfusion Rats," BioMed Research International, vol. 2018, Article ID 5423961, 8 pages, 2018.

[70] M. H. Shen, Q. Q. Tang, Z. R. Li, and C. Ma, "Effect of electroacupuncture on hippocampal LTP in Alzheimer' s disease rats induced by Abeta(25-35)," Zhen Ci Yan Jiu, vol. 35, no. 1, pp. 3-7, 2010.

[71] P. Greengard, F. Valtorta, A. J. Czernik, and F. Benfenati, "Synaptic vesicle phosphoproteins and regulation of synaptic function," Science, vol. 259, no. 5096, pp. 780-785, 1993.

[72] A. Citri and R. C. Malenka, "Synaptic plasticity: multiple forms, functions, and mechanisms," Neuropsychopharmacology, vol. 33, no. 1, pp. 18-41, 2008.

[73] S. B. Martin, A. L. S. Dowling, J. Lianekhammy et al., "Synaptophysin and synaptojanin-1 in Down syndrome are differentially affected by Alzheimer's disease," Journal of Alzheimer's Disease, vol. 42, no. 3, pp. 767-775, 2014.

[74] J. Dong, J. Zhao, Y. Lin et al., "Exercise improves recognition memory and synaptic plasticity in the prefrontal cortex for rats modelling vascular dementia," Neurological Research, vol. 40, pp. 68-77, 2017.

[75] G. Yang, Y.-N. Pei, S.-J. Shao et al., "Effects of electroacupuncture at "Baihui" and "Yongquan" on the levels of synaptic plasticity related proteins postsynaptic density-95 and synaptophysin in hippocampus of APP/PS1 mice," Zhen Ci Yan Jiu, vol. 45, no. 4, pp. 310-314, 2020.

[76] W. Dong, W. Yang, F. Li et al., "Electroacupuncture improves synaptic function in SAMP8 mice probably via inhibition of the AMPK/eEF2K/eEF2 signaling pathway," Evidence-based Complementary and Alternative Medicine, vol. 2019, Article ID 8260815, 10 pages, 2019.

[77] Y. Wang, L. Kong, W. Li et al., "Effects and mechanisms of different frequencies of electroacupuncture for learning and 
memory ability of Alzheimer's rats," Zhongguo Zhen Jiu, vol. 37, no. 6, pp. 629-636, 2017.

[78] X. He, T. Yan, R. Chen, and D. Ran, "Acute effects of electroacupuncture (EA) on hippocampal long term potentiation (LTP) of perforant path-dentate gyrus granule cells synapse related to memory," Acupunct Electrother Res, vol. 37, no. 2, pp. 89-101, 2012.

[79] N. A. Otmakhova and J. E. Lisman, "D1/D5 dopamine receptor activation increases the magnitude of early long-term potentiation at CA1 hippocampal synapses," The Journal of Neuroscience, vol. 16, no. 23, pp. 7478-7486, 1996.

[80] N. X. Tritsch and B. L. Sabatini, "Dopaminergic modulation of synaptic transmission in cortex and striatum," Neuron, vol. 76, no. 1, pp. 33-50, 2012.

[81] B. Lee, B. Sur, J. Shim, D. H. Hahm, and H. Lee, "Acupuncture stimulation improves scopolamine-induced cognitive impairment via activation of cholinergic system and regulation of BDNF and CREB expressions in rats," BMC Complementary and Alternative Medicine, vol. 14, no. 1, p. 338, 2014.

[82] F. Arima-Yoshida, A. M. Watabe, and T. Manabe, "The mechanisms of the strong inhibitory modulation of longterm potentiation in the rat dentate gyrus," The European Journal of Neuroscience, vol. 33, no. 9, pp. 1637-1646, 2011.

[83] P. C. Kind and P. E. Neumann, "Plasticity: downstream of glutamate," Trends in Neurosciences, vol. 24, no. 10, pp. 553-555, 2001.

[84] L. Liu, T. P. Wong, M. F. Pozza et al., "Role of NMDA receptor subtypes in governing the direction of hippocampal synaptic plasticity," Science, vol. 304, no. 5673, pp. 1021-1024, 2004.

[85] T. E. Bartlett, N. J. Bannister, V. J. Collett et al., "Differential roles of NR2A and NR2B-containing NMDA receptors in LTP and LTD in the CA1 region of two-week old rat hippocampus," Neuropharmacology, vol. 52, no. 1, pp. 60-70, 2007.

[86] Y. Zhang, X. Mao, R. Lin, Z. Li, and J. Lin, "Electroacupuncture ameliorates cognitive impairment through inhibition of $\mathrm{Ca}^{2+}$-mediated neurotoxicity in a rat model of cerebral ischaemia-reperfusion injury," Acupuncture in Medicine, vol. 36, no. 6, pp. 401-407, 2018.

[87] Y. Mu, S. W. Lee, and F. H. Gage, "Signaling in adult neurogenesis," Current Opinion in Neurobiology, vol. 20, no. 4, pp. 416-423, 2010.

[88] T. Iso, L. Kedes, and Y. Hamamori, "HES and HERP families: multiple effectors of the Notch signaling pathway," Journal of Cellular Physiology, vol. 194, no. 3, pp. 237-255, 2003.

[89] H.-d. Guo, J.-x. Tian, J. Zhu et al., "Electroacupuncture suppressed neuronal apoptosis and improved cognitive impairment in the AD model rats possibly via downregulation of Notch signaling pathway," Evidence-based Complementary and Alternative Medicine, vol. 2015, Article ID 393569, 9 pages, 2015.

[90] D. K. Binder and H. E. Scharfman, "Brain-derived neurotrophic factor," Growth Factors, vol. 22, pp. 123-131, 2009.

[91] M. F. Egan, M. Kojima, J. H. Callicott et al., "The BDNF val66met polymorphism affects activity-dependent secretion of BDNF and human memory and hippocampal function," Cell, vol. 112, pp. 257-269, 2003.

[92] Z. Y. Chen, P. D. Patel, G. Sant et al., "Variant brain-derived neurotrophic factor (BDNF) (Met66) alters the intracellular trafficking and activity-dependent secretion of wild-type
BDNF in neurosecretory cells and cortical neurons," The Journal of Neuroscience, vol. 24, no. 18, pp. 4401-4411, 2004.

[93] P. T. Pang, H. K. Teng, E. Zaitsev et al., "Cleavage of proBDNF by $\mathrm{tPA}$ /plasmin is essential for long-term hippocampal plasticity," Science, vol. 306, no. 5695, pp. 487-491, 2004.

[94] B. Lu, P. T. Pang, and N. H. Woo, "The yin and yang of neurotrophin action," Nature Reviews. Neuroscience, vol. 6, no. 8, pp. 603-614, 2005.

[95] R. Lee, P. Kermani, K. K. Teng, and B. L. Hempstead, "Regulation of cell survival by secreted proneurotrophins," Science, vol. 294, no. 5548, pp. 1945-1948, 2001.

[96] R. Lin, J. Chen, X. Li et al., "Electroacupuncture at the Baihui acupoint alleviates cognitive impairment and exerts neuroprotective effects by modulating the expression and processing of brain-derived neurotrophic factor in APP/PS1 transgenic mice," Molecular Medicine Reports, vol. 13, no. 2, pp. 1611-1617, 2016.

[97] R. Lin, L. Li, Y. Zhang et al., "Electroacupuncture ameliorate learning and memory by improving $\mathrm{N}$-acetylaspartate and glutamate metabolism in APP/PS1 mice," Biological Research, vol. 51, no. 1, p. 21, 2018.

[98] J. Xiong, Z. Zhang, Y. Ma et al., “The effect of combined scalp acupuncture and cognitive training in patients with stroke on cognitive and motor functions," NeuroRehabilitation, vol. 46, pp. 75-82, 2020.

[99] Z. C. Hesp, E. Z. Goldstein, C. J. Miranda, B. K. Kaspar, and D. M. McTigue, "Chronic oligodendrogenesis and remyelination after spinal cord injury in mice and rats," The Journal of Neuroscience, vol. 35, no. 3, pp. 1274-1290, 2015.

[100] T. Arendt, J. T. Stieler, and M. Holzer, "Tau and tauopathies," Brain Research Bulletin, vol. 126, pp. 238-292, 2016.

[101] J. Jiang, G. Liu, S. Shi, Y. Li, and Z. Li, "Effects of manual acupuncture combined with donepezil in a mouse model of Alzheimer's disease," Acupuncture in Medicine, vol. 37, no. 1, pp. 64-71, 2019.

[102] P. H. Reddy, "Amyloid beta-induced glycogen synthase kinase $3 \beta$ phosphorylated VDAC1 in Alzheimer's disease: implications for synaptic dysfunction and neuronal damage," Biochimica et Biophysica Acta-Molecular Basis of Disease, vol. 1832, no. 12, pp. 1913-1921, 2013.

[103] L. Avrahami, D. Farfara, M. Shaham-Kol, R. Vassar, D. Frenkel, and H. Eldar-Finkelman, "Inhibition of glycogen synthase kinase-3 ameliorates $\beta$-amyloid pathology and restores lysosomal acidification and mammalian target of rapamycin activity in the Alzheimer disease mouse model: in vivo and in vitro studies," The Journal of Biological Chemistry, vol. 288, no. 2, pp. 1295-1306, 2013.

[104] S. N. Duffy, K. J. Craddock, T. Abel, and P. V. Nguyen, "Environmental enrichment modifies the PKA-dependence of hippocampal LTP and improves hippocampus-dependent memory," Learning \& Memory, vol. 8, no. 1, pp. 26-34, 2001.

[105] S. J. Raiker, H. Lee, K. T. Baldwin, Y. Duan, P. Shrager, and R. J. Giger, "Oligodendrocyte-myelin glycoprotein and Nogo negatively regulate activity-dependent synaptic plasticity," The Journal of Neuroscience, vol. 30, pp. 12432-12445, 2010.

[106] L. Sui, J. Wang, and B. M. Li, "Role of the phosphoinositide 3kinase-Akt-mammalian target of the rapamycin signaling pathway in long-term potentiation and trace fear conditioning memory in rat medial prefrontal cortex," Learning \& Memory, vol. 15, no. 10, pp. 762-776, 2008. 
[107] C. A. Hoeffer, W. Tang, H. Wong et al., "Removal of FKBP12 enhances mTOR-Raptor interactions, LTP, memory, and perseverative/repetitive behavior," Neuron, vol. 60, no. 5, pp. 832-845, 2008.

[108] L. Swiech, M. Perycz, A. Malik, and J. Jaworski, "Role of mTOR in physiology and pathology of the nervous system," Biochimica et Biophysica Acta, vol. 1784, no. 1, pp. 116-132, 2008.

[109] Y. Zhu, Y. Zeng, X. Wang, and X. Ye, "Effect of electroacupuncture on the expression of mTOR and eIF4E in hippocampus of rats with vascular dementia," Neurological Sciences, vol. 34, no. 7, pp. 1093-1097, 2013.

[110] T. Fukada and N. K. Tonks, "Identification of YB-1 as a regulator of PTP1B expression: implications for regulation of insulin and cytokine signaling," The EMBO Journal, vol. 22, no. 3, pp. 479-493, 2003.

[111] L. Sang, C. Liu, L. Wang et al., "Disrupted brain structural connectivity network in subcortical ischemic vascular cognitive impairment with no dementia," Frontiers in Aging Neuroscience, vol. 12, p. 6, 2020.

[112] R. A. Sperling, P. S. LaViolette, K. O'Keefe et al., "Amyloid deposition is associated with impaired default network function in older persons without dementia," Neuron, vol. 63, no. 2, pp. 178-188, 2009.

[113] Z. Wang, B. Nie, D. Li et al., "Effect of acupuncture in mild cognitive impairment and Alzheimer disease: a functional MRI study,” PLoS One, vol. 7, no. 8, article e42730, 2012.

[114] Y. Tang, Y. Xing, Z. Zhu et al., "The effects of 7-week cognitive training in patients with vascular cognitive impairment, no dementia (the Cog-VACCINE study): a randomized controlled trial," Alzheimers Dement, vol. 15, no. 5, pp. 605-614, 2019.

[115] M. Stampanoni Bassi, E. Iezzi, L. Gilio, D. Centonze, and F. Buttari, "Synaptic plasticity shapes brain connectivity: implications for network topology," International Journal of Molecular Sciences, vol. 20, no. 24, p. 6193, 2019.

[116] C.-c. Yu, C.-y. Ma, H. Wang et al., "Effects of acupuncture on Alzheimer's disease: evidence from neuroimaging studies," Chinese Journal of Integrative Medicine, vol. 25, no. 8, pp. 631-640, 2019.

[117] W. Zheng, Z. Su, X. Liu et al., "Modulation of functional activity and connectivity by acupuncture in patients with Alzheimer disease as measured by resting-state fMRI," PLoS One, vol. 13, article e0196933, 2018.

[118] Y. Shan, J.-J. Wang, Z.-Q. Wang et al., "Neuronal specificity of acupuncture in Alzheimer's disease and mild cognitive impairment patients: a functional MRI study," Evidencebased Complementary and Alternative Medicine, vol. 2018, Article ID 7619197, 10 pages, 2018.

[119] Y. Feng, L. Bai, Y. Ren et al., "FMRI connectivity analysis of acupuncture effects on the whole brain network in mild cognitive impairment patients," Magnetic Resonance Imaging, vol. 30, no. 5, pp. 672-682, 2012.

[120] L. Zhao, F. Zhu, S. Chen et al., "Acupuncture at the Taixi (KI3) acupoint activates cerebral neurons in elderly patients with mild cognitive impairment," Neural Regeneration Research, vol. 9, pp. 1163-1168, 2014.

[121] S. Chen, L. Bai, M. Xu et al., "Multivariate granger causality analysis of acupuncture effects in mild cognitive impairment patients: an FMRI study," Evidence-based Complementary and Alternative Medicine, vol. 2013, Article ID 127271, 12 pages, 2013.

[122] P. Fu, J. P. Jia, J. Zhu, and J. J. Huang, "Effects of acupuncture at Neiguan (PC 6) on human brain functional imaging in different functional states," Zhongguo Zhen Jiu, vol. 25, pp. 784-786, 2005.

[123] Y. Zhou and J. Jia, "Effect of acupuncture given at the HT 7, ST 36, ST 40 and KI 3 acupoints on various parts of the brains of Alzheimer's disease patients," Acupuncture \& ElectroTherapeutics Research, vol. 33, no. 1, pp. 9-17, 2008.

[124] T. T. Tan, D. Wang, J. K. Huang et al., "Modulatory effects of acupuncture on brain networks in mild cognitive impairment patients," Neural Regeneration Research, vol. 12, no. 2, pp. 250-258, 2017.

[125] H. Li, Z. Wang, H. Yu et al., "The long-term effects of acupuncture on hippocampal functional connectivity in aMCI with hippocampal atrophy: a randomized longitudinal fMRI study," Neural Plasticity, vol. 2020, 9 pages, 2020.

[126] L. Bai, M. Zhang, S. Chen et al., "Characterizing acupuncture de qi in mild cognitive impairment: relations with smallworld efficiency of functional brain networks," Evidencebased Complementary and Alternative Medicine, vol. 2013, Article ID 304804, 8 pages, 2013.

[127] L. Y. Xiao, X. R. Wang, Y. Yang et al., "Applications of acupuncture therapy in modulating plasticity of central nervous system," Neuromodulation, vol. 21, no. 8, pp. 762-776, 2018.

[128] N. Ding, J. Jiang, A. Xu, Y. Tang, and Z. Li, "Manual acupuncture regulates behavior and cerebral blood flow in the SAMP8 mouse model of Alzheimer's disease," Frontiers in Neuroscience, vol. 13, p. 37, 2019.

[129] J. Kong, R. Gollub, T. Huang et al., "Acupuncture de qi, from qualitative history to quantitative measurement," Journal of Alternative and Complementary Medicine, vol. 13, no. 10, pp. 1059-1070, 2007. 